\title{
Disorder-induced noncollinear ferromagnetism in models for (III,Mn)V semiconductors
}

\author{
John Schliemann \\ Department of Physics and Astronomy, University of Basel, CH-4056 Basel, Switzerland \\ and Department of Physics, The University of Texas, Austin, Texas 78712
}

(Received 30 September 2002; published 24 January 2003)

\begin{abstract}
We study the ground-state properties of kinetic-exchange models for (III,Mn)V semiconductors with randomly distributed Mn ions. Our method is embedded in a path integral spin-wave-type formalism leading to an effective action for Mn spins only with full Matsubara frequency dependence. The zero-frequency contribution to this action is equivalent to static perturbation theory and characterizes the stability of a given spin configuration, while the component linear in frequency can be interpreted as the joint Berry phase of the Mn and carrier system. For simple parabolic-band carriers the collinear ferromagnetic state with all Mn spins in parallel is always stationary but generically unstable. This instability can be characterized in terms of inverse participation ratios and is due to long-ranged nonlocal spin fluctuations. We also present results for the ground-state magnetization as a function of an external field. For carrier dispersions involving anisotropy induced by spin-orbit coupling the collinear state is not even stationary and therefore also not the ground state. This interplay between the anisotropy in the carrier system and the disorder in the Mn positions reflects recent findings by Zarand and Janko [Phys. Rev. Lett. 89, 047201 (2002)] obtained within the RKKY approximation. The stationarity of the collinear state (with the magnetization pointing in one of the cubic symmetry directions) is restored in the continuum or virtual crystal approximation where disorder is neglected.
\end{abstract}

DOI: 10.1103/PhysRevB.67.045202

PACS number(s): 75.50.Pp, 75.30.Ds, 75.50.Lk

\section{INTRODUCTION}

For several years, diluted ferromagnetic semiconductors have been in the focus of research in solid-state physics; for reviews, see Refs. 1-8. This is, on the one hand, because these systems provide interesting and challenging physical problems on their own right. On the other hand, this great deal of interest is due to possible applications of these materials in the emerging field of spintronics ${ }^{9,10}$ since they offer the perspective of combining ferromagnetism with the readily tunable transport properties of semiconductors.

An important achievement in this field was the fabrication of diluted Mn-doped GaAs via low-temperature molecular beam epitaxy by Ohno and collaborators in $1996 .^{11}$ This material showed a Curie temperature of $110 \mathrm{~K}$, a result which has been reproduced in the meantime by several other groups. Moreover, very recently reports on Mn-doped (III,V) semiconductors having Curie temperatures of room temperature or higher have appeared, and also other combinations of magnetic ions and/or host materials look prospective. ${ }^{12-19}$

These recent developments have also already generated a large amount of theoretical research on diluted ferromagnetic semiconductors. ${ }^{5-8,20-53}$ Such studies include electronic structure calculations based on density functional techniques ${ }^{8,51-53}$ and investigations using specific models for such systems. ${ }^{5-7,20-50}$ In particular, very recently a series of studies has appeared on disorder-related phenomena in different models for diluted ferromagnetic semiconductors. $^{29-32,36-48}$ In the present work we employ kinetic-exchange models for carrier-mediated ferromagnetism occurring in Mn-doped (III,V) semiconductors which have been the basis of a large body of previous work. ${ }^{5,20-34}$ The general Hamiltonian reads

$$
\mathcal{H}=\mathcal{H}_{\text {kin }}+\sum_{I} \int d^{3} r J\left(\vec{r}-\vec{R}_{I}\right) \vec{s}(\vec{r}) \cdot \vec{S}_{I}
$$

These models show several features in accordance with experiments. They consist of a kinetic term $\mathcal{H}_{\text {kin }}$ for free valence-band carriers (holes) whose spin density $\vec{s}(\vec{r})$ is antiferromagnetically exchange coupled to localized Mn spins $\vec{S}_{I}$ of length $S=5 / 2$ at locations $\vec{R}_{I}$ by an spatially extended coupling $J(\vec{r})$, which we take to be of the form

$$
J(\vec{r})=\frac{J_{p d}}{\left(2 \pi a_{0}^{2}\right)^{3 / 2}} e^{-r^{2} / 2 a_{0}^{2}}
$$

The regularization parameter $a_{0}$ reflects the spatial range of the exchange coupling. ${ }^{46}$ In a minimal description of carrierinduced ferromagnetism the kinetic term represents just a simple parabolic band characterized by an effective mass $m^{*}$. Moreover, we will also consider the case of a more realistic $\vec{k} \cdot \vec{p}$ Hamiltonian ${ }^{26,54,55}$ describing the valence-band structure of (III,V) semiconductors such as GaAs.

Differently from earlier work ${ }^{20-27,31}$ we will not make use of the virtual crystal approximation; i.e., we will not approximate the localized Mn moments by a continuum but retain them as individual and randomly distributed spins. This additional feature of the model grossly enriches its physical properties and gives rise to the occurrence of noncollinear ferromagnetism. ${ }^{30}$ This noncollinearity in the orientations of localized magnetic moments even in the ground state of the system is very likely to be an ingredient in the interpretation of recent experiments by Potashnik et al. ${ }^{56}$ who found a strong dependence of the magnetic properties of Mn-doped GaAs on the annealing history of the sample. Similarly strong dependences are also found in transport measurements ${ }^{56}$ and in crystallographic properties. ${ }^{57}$

Our theoretical method and results to be presented here can be embedded in a path integral approach to the partition function of the underlying model. A part of the results was 
already discussed in Refs. 5 and 30. In the present paper we add further details to the formalism and present new results on the frequency dependence of the action kernel and the role of an external magnetic field.

Moreover, we give a detailed discussion of the gradient of the carrier ground-state energy with respect to fluctuations in the orientations of the localized Mn moments. For the case of isotropic systems with simple parabolic-band carriers the collinear ferromagnetic state with all Mn spins in parallel is always stationary (i.e., it has a vanishing energy gradient), but in general not stable..$^{30}$ In the present work we show the possibility of complanar spin structures which are local minima on the energy landscape of such systems. For the case of models with spin-orbit anisotropy in the carrier system we find that the collinear state is not even stationary and does therefore not represent an energy minimum. This finding resembles recent results by Zarand and Janko. ${ }^{40}$

Another study related to the present one was reported on recently by Korzhavyi et al. ${ }^{48}$ These authors investigate, by both experimental and theoretical means, the influence of As antisite defects on the ground-state structure of the Mn local magnetic moments. As a result, As antisite defects are found to favor the formation of disordered local moment configurations of $\mathrm{Mn}$ spins with antiparallel orientation and therefore reduce the net magnetization. However, differently from the results of the present investigations, the Mn spins in the disordered local moment configurations are restricted to have (at random) either spin up or down with respect to some given quantization axis; i.e., they are collinear to each other.

This paper is organized as follows. In Sec. II we review our general theory of magnetic fluctuations in the kinetic exchange models studied here and add further technical details. In Sec. III we report on numerical results concerning the instability of the collinear ferromagnetic state in the parabolic-band model. In Sec. IV we discuss several results arising from the very general gradient expression for the ground-state energy obtained in Sec. II. In Sec. IV A 1 we demonstrate the occurrence of complanar or helical energy minima in the parabolic-band model. An important further finding is discussed in Sec. IV A 2 where the collinear state is shown not even to be stationary with respect to magnetic fluctuations if anisotropy induced by spin-orbit coupling is present. In Sec. IV B we report on numerical steepestdescent studies of the true energy minima below the collinear state and on the influence of an external magnetic field. In Sec. V we discuss the small-frequency dependence of the effective-action kernel derived in Sec. II. We close with conclusions in Sec. VI.

\section{MAGNETIC FLUCTUATIONS: GENERAL THEORY}

\section{A. Path integral approach}

We now describe a path integral approach to the partition function of fluctuations around a given magnetic state of the Mn spins. Our method is similar to the one used in Ref. 25 with the differences that we do not approximate the Mn magnetic moments by a continuum but retain them as individual localized spins, and we study fluctuations around more general noncollinear magnetic states. The state we consider is a tensor product of spin-coherent states for each Mn location $I$ with a polarization direction $\vec{\Omega}_{I}$ $=\left(\sin \vartheta_{I} \cos \varphi_{I}, \sin \vartheta_{I} \sin \varphi_{I}, \cos \vartheta_{I}\right)$. We introduce local coordinates for each $\mathrm{Mn}$ site whose $\hat{z}$ direction coincides with $\vec{\Omega}_{I}$. The spin operators $\vec{T}_{I}$ with respect to these local bases are related to the original operators $\vec{S}_{I}$ via

$$
\begin{aligned}
\left(\begin{array}{c}
S_{I}^{x} \\
S_{I}^{y} \\
S_{I}^{z}
\end{array}\right) & =\left(\begin{array}{ccc}
\cos \varphi_{I} & -\sin \varphi_{I} & 0 \\
\sin \varphi_{I} & \cos \varphi_{I} & 0 \\
0 & 0 & 1
\end{array}\right) \\
& \times\left(\begin{array}{ccc}
\cos \vartheta_{I} & 0 & \sin \vartheta_{I} \\
0 & 1 & 0 \\
-\sin \vartheta_{I} & 0 & \cos \vartheta_{I}
\end{array}\right)\left(\begin{array}{c}
T_{I}^{x} \\
T_{I}^{y} \\
T_{I}^{z}
\end{array}\right) .
\end{aligned}
$$

To parametrize fluctuations around the magnetic state given by the directions $\vec{\Omega}_{I}$ we use the usual Holstein-Primakoff representation of the spin operators $\vec{T}_{I}$,

$$
T^{+}=\sqrt{2 S-b_{I}^{+} b_{I}} b_{I}, \quad T^{z}=S-b_{I}^{+} b_{I}
$$

Formulating the partition function as a path integral and representing the Mn spin Holstein-Primakoff bosons in a coherent-state parameterization one arrives after integrating out the carrier degrees of freedom at the following effective action:

$$
S_{\mathrm{eff}}=\int_{0}^{\beta} d \tau \sum_{I}\left[\bar{z}_{I} \partial_{\tau} z_{I}\right]-\operatorname{tr}\left[\ln \left(G_{M F}^{-1}+\delta G^{-1}\right)\right] .
$$

$z_{I}(\tau)$ stands for the bosonic Holstein-Primakoff field parametrizing the fluctuations of the $\mathrm{Mn}$ spin $I$ around its mean direction $\vec{\Omega}_{I}$. The integration over the imaginary time $\tau$ goes from zero to the inverse temperature $\beta$, and the trace in the second contribution is over fermionic carrier degrees of freedom and imaginary time. The the fluctuation-free (i.e., meanfield) part $G_{M F}^{-1}$ of the integral kernel is given by

$$
\begin{aligned}
& G_{M F}^{-1}=\partial_{\tau}-\mu-\mathcal{H}_{k i n}+S \sum_{I} J\left(\vec{r}-\vec{R}_{I}\right)\left[\cos \vartheta_{I} s^{z}\right.
\end{aligned}
$$

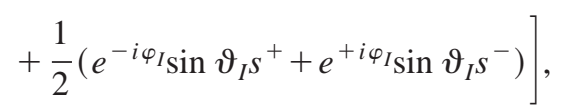

where $\mu$ is a chemical potential and $s^{z}$ and $s^{ \pm}=s^{x} \pm i s^{y}$ are carrier spin operators. In the case of a simple parabolic band they are just proportional to Pauli matrices while, for instance, in the case of a six-band $\vec{k} \cdot \vec{p}$ Hamiltonian they have a more complex form. ${ }^{26,54}$

The fluctuation part of the inverse Green's function in up to quadratic order in the Holstein-Primakoff variables reads $\delta G^{-1}=\delta G_{1}^{-1}+\delta G_{2}^{-1}$ with 


$$
\begin{aligned}
\delta G_{1}^{-1}= & \frac{1}{2} \sum_{I} J\left(\vec{r}-\vec{R}_{I}\right)\left[e^{-i \varphi_{I}} \sqrt{2 S}\left(\bar{z}_{I} \cos ^{2} \frac{\vartheta_{I}}{2}-z_{I} \sin ^{2} \frac{\vartheta_{I}}{2}\right) s^{+}\right. \\
& +e^{+i \varphi_{I}} \sqrt{2 S}\left(z_{I} \cos ^{2} \frac{\vartheta_{I}}{2}-\bar{z}_{I} \sin ^{2} \frac{\vartheta_{I}}{2}\right) s^{-} \\
& \left.-\sqrt{2 S} \sin \vartheta_{I}\left(z_{I}+\bar{z}_{I}\right) s^{z}\right] \\
\delta G_{2}^{-1}= & -\sum_{I} J\left(\vec{r}-\vec{R}_{I}\right)\left[z_{I} \bar{z}_{I} \cos \vartheta_{I} s^{z}+\frac{1}{2} z_{I} \bar{z}_{I} \sin \vartheta_{I}\right. \\
& \left.\times\left(e^{-i \varphi_{I} S^{+}}+e^{+i \varphi_{I} S^{-}}\right)\right] .
\end{aligned}
$$

To analyze magnetic fluctuations we expand the action (5) in the bosonic spin variables describing deviations from the prescribed directions $\vec{\Omega}_{I}$,

$$
\operatorname{tr} \ln \left(G_{M F}^{-1}+\delta G^{-1}\right)=\operatorname{tr} \ln \left(G_{M F}\right)-\sum_{n=1}^{\infty} \frac{1}{n} \operatorname{tr}\left(-G_{M F} \delta G^{-1}\right)^{n} .
$$

We will evaluate the effective action in up to second order in the fluctuations $z_{I}(\tau)$. To this end we introduce the Fourier transforms $z_{I}\left(\Omega_{n}\right)=\int_{0}^{\beta} d \tau \exp \left(i \Omega_{n} \tau\right) z_{I}(\tau)$ with the notation $\bar{z}_{I}\left(\Omega_{n}\right)=\overline{z_{I}\left(\Omega_{n}\right)}$, where $\Omega_{n}=2 n \pi / \beta, n$ integer, is a bosonic Matsubara frequency.

In this subsection we shall consider a simple parabolicband model where the fluctuation-free part of the carrier Green's function reads in real-space representation

$$
G_{M F}\left(\vec{r}, \sigma ; \vec{r}^{\prime}, \sigma^{\prime} ; \omega_{n}\right)=-\sum_{\alpha} \frac{\psi_{\alpha \sigma}(\vec{r}) \bar{\psi}_{\alpha \sigma^{\prime}}\left(\vec{r}^{\prime}\right)}{i \omega_{n}-\eta_{\alpha}} .
$$

Here $\omega_{n}=(2 n+1) \pi / \beta, n$ integer, is a fermionic Matsubara frequency and $\psi_{\alpha \sigma}(\vec{r})$ is the spin component $\sigma$ of the carrier wave function with label $\alpha$ and energy $\varepsilon_{\alpha}=\eta_{\alpha}+\mu$. These wave functions are the eigenstates of the single-particle mean-field Hamiltonian $\mathcal{H}_{M F}=G_{M F}^{-1}-\partial_{\tau}+\mu$.

The lowest-order contribution in the effective action occurs at zero Matsubara frequency only and is linear in the Holstein-Primakoff variables:

$$
\mathcal{S}_{\text {fluc }}^{(1)}=\frac{1}{2} \sum_{I}\left[\bar{g}_{I} z_{I}(0)+g_{I} \bar{z}_{I}(0)\right],
$$

with $g_{I}=g_{I}^{1}+i g_{I}^{2}$ and

$$
\begin{aligned}
g_{I}^{1}= & \sqrt{2 S}\left(\vec{e}_{\varphi_{I}} \times \vec{e}_{z}\right) \cdot \int d^{3} r\left(J ( \vec { r } - \vec { R } _ { I } ) \left\{\left[\langle\vec{s}(\vec{r})\rangle \cdot \vec{e}_{\varphi_{I}}\right] \vec{e}_{\varphi_{I}}\right.\right. \\
& \left.\left.+\left[\langle\vec{s}(\vec{r})\rangle \cdot \vec{e}_{z}\right] \vec{e}_{z}\right\} \times \vec{\Omega}_{I}\right), \\
& g_{I}^{2}=\sqrt{2 S} \vec{e}_{z} \cdot\left(\vec{e}_{\varphi_{I}} \times \int d^{3} r J\left(\vec{r}-\vec{R}_{I}\right)\langle\vec{s}(\vec{r})\rangle\right) .
\end{aligned}
$$

Here $\langle\vec{s}(\vec{r})\rangle$ is the expectation value of the carrier spin density, $\vec{e}_{\varphi_{I}}=\left(\cos \varphi_{I}, \sin \varphi_{I}, 0\right)$ and $\vec{e}_{z}=(0,0,1)$.
The contribution $S_{\text {fluc }}^{(2)}$ bilinear in the Holstein-Primakoff variables is quite complex for general $\mathrm{Mn}$ spin orientations $\Omega_{I}$. In particular, numerical evaluations of this quantity are extraordinarily tedious and computationally expensive. We therefore shall concentrate on the collinear state where all Mn spins point, say, along the $z$-direction. Then the secondorder contribution to the effective action reads

$$
S_{\text {fluc }}^{(2)}=\frac{1}{\beta} \sum_{n} \sum_{I, J} \bar{z}_{I}\left(\Omega_{n}\right) D_{I J}^{-1}\left(\Omega_{n}\right) z_{J}\left(\Omega_{n}\right)
$$

where the fluctuation matrix $D_{I J}^{-1}\left(\Omega_{n}\right)$ reads

$$
D_{I J}^{-1}\left(\Omega_{n}\right)=L_{I J}\left(\Omega_{n}\right)+K_{I J}\left(\Omega_{n}\right)
$$

with

$$
\begin{aligned}
L_{I J} & =\delta_{I J}\left(-i \Omega_{n}-\int d^{3} r J\left(\vec{r}-\vec{R}_{I}\right)\left\langle s^{z}(\vec{r})\right\rangle\right), \\
K_{I J} & =\frac{S}{2} \sum_{\alpha, \beta}\left[\frac{n_{F}\left(\eta_{\alpha}\right)-n_{F}\left(\eta_{\beta}\right)}{i \Omega_{n}+\eta_{\alpha}-\eta_{\beta}} F_{I}^{\alpha \downarrow, \beta \uparrow} F_{J}^{\beta \uparrow, \alpha \downarrow}\right] .
\end{aligned}
$$

Here $n_{F}$ is the Fermi function and

$$
F_{I}^{\alpha \sigma, \beta \mu}=\int d^{3} r J\left(\vec{r}-\vec{R}_{I}\right) \bar{\psi}_{\alpha \sigma}(\vec{r}) \psi_{\beta \mu}(\vec{r}) .
$$

All quantities referring to the carrier system are to be evaluated for the collinear orientation of Mn spins.

The diagonal contributions to the action kernel summarized in $L_{I J}$ stem from the kinetic term $\Sigma_{I} \bar{z}_{I} \partial_{\tau} z_{I}$ in the integrand of Eq. (5) and from the lowest-order term $\operatorname{tr}\left(G_{M F} \delta G_{2}^{-1}\right)$ in Eq. (9). The term given by $K_{I J}$ arises from the bubble contribution $-\left[\operatorname{tr}\left(G_{M F} \delta G_{1}^{-1} G_{M F} \delta G_{1}^{-1}\right)\right] / 2$.

\section{B. Static limit: Perturbation theory}

The limit of zero Matsubara frequency $\Omega_{n}$ corresponds to static perturbations of the mean-field carrier ground state. It is instructive to verify this explicitly by elementary perturbation theory. The unperturbed mean-field Hamiltonian is again $\mathcal{H}_{M F}=G_{M F}^{-1}-\partial_{\tau}+\mu$ which is subject to a perturbation $\mathcal{H}_{\text {fluc }}\left[\left\{z_{I}, \bar{z}_{I}\right\}\right]$ with

$$
\mathcal{H}_{\text {fluc }}\left[\left\{z_{I}, \bar{z}_{I}\right\}\right]=\delta G_{1}^{-1}\left[\left\{z_{I}, \bar{z}_{I}\right\}\right]+\delta G_{2}^{-1}\left[\left\{z_{I}, \bar{z}_{I}\right\}\right] .
$$

Here $\delta G_{1}^{-1}$ and $\delta G_{2}^{-1}$ have the same form as in Eqs. (7) and (8) with $z_{I}=\left(T^{x}+i T^{y}\right) / \sqrt{2 S}$ parametrizing the perturbations with respect to the prescribed general directions $\vec{\Omega}_{I}$ in $\mathcal{H}_{M F}$.

We now consider the contributions from $\mathcal{H}_{\text {fluc }}$ to the energy of the carrier ground state. In linear order in $z_{I}$ one has the contribution from the expectation value of $\delta G_{1}^{-1}$ which can be written as

$$
E^{(1)}=\frac{1}{2} \sum_{I}\left[\bar{g}_{I} z_{I}+g_{I} \bar{z}_{I}\right]
$$

with $g_{I}=g_{I}^{1}+i g_{I}^{2}$ given by Eqs. (12) and (13). This expression coincides with Eq. (11) ${ }^{58}$ The expectation values of the 
carrier spin density $\langle\vec{s}(\vec{r})\rangle$ entering Eq. (20) via the complex coefficients $g_{I}$ have to be computed within the ground-state of the unperturbed Hamiltonian $\mathcal{H}_{M F}$. This operator depends on the Mn spin directions $\vec{\Omega}_{I}$ and on the kinetic term $\mathcal{H}_{\text {kin }}$ for the carriers, which has not been specified so far within this perturbational approach to the ground state energy. Therefore the expression (20) holds formally for any band representation of the carrier system. In particular, Eq. (20) is valid for the simple parabolic-band model as well as for more sophisticated $\vec{k} \cdot \vec{p}$ Hamiltonians. We will come back to this important fact in Sec. III A.

There are two contribution bilinear in $z_{I}$. The first one is just the expectation value of $\delta G_{2}^{-1}$ within the carrier ground state,

$$
E_{L}^{(2)}=\sum_{I, J} \bar{z}_{I} L_{I J} z_{J}
$$

with

$$
L_{I J}=-\delta_{I J} \int d^{3} r J\left(\vec{r}-\vec{R}_{I}\right)\langle\vec{s}(\vec{r})\rangle \vec{\Omega}_{I},
$$

which obviously coincides with Eq. (16) for $\Omega_{n}=0$ and $\vec{\Omega}_{I}$ $=\vec{e}_{z}$ for all $I$. The other bilinear contribution is the secondorder term arising from $\delta G_{1}^{-1}$ which reads

$$
\begin{aligned}
E_{K}^{(2)}= & \frac{S}{4} \sum_{\alpha, \beta}\left[\frac{n_{F}\left(\eta_{\alpha}\right)-n_{F}\left(\eta_{\beta}\right)}{\eta_{\alpha}-\eta_{\beta}} \mid \sum_{I}\left[\operatorname { R e } \{ z _ { I } \} \operatorname { s i n } \vartheta _ { I } \left(F^{\alpha \downarrow, \beta \downarrow}\right.\right.\right. \\
& \left.-F^{\alpha \uparrow, \beta \uparrow}\right)+\left(\cos \vartheta_{I} \operatorname{Re}\left\{z_{I}\right\}-i \operatorname{Im}\left\{z_{I}\right\}\right) F^{\alpha \uparrow, \beta \downarrow} e^{-i \varphi_{I}} \\
& \left.+\left.\left(\cos \vartheta_{I} \operatorname{Re}\left\{z_{I}\right\}+i \operatorname{Im}\left\{z_{I}\right\}\right) F^{\alpha \downarrow, \beta \uparrow} e^{\left.+i \varphi_{I}\right]}\right|^{2}\right]
\end{aligned}
$$

It is straightforward to see that in the collinear case, $\vec{\Omega}_{I}$ $=\vec{e}_{z}$ for all $I$, this expression takes the form

$$
E_{K}^{(2)}=\sum_{I, J} \bar{z}_{I} K_{I J} z_{J}
$$

with $K_{I J}=K_{I J}\left(\Omega_{n}=0\right)$ given by Eq. (17). ${ }^{59}$

We note that the perturbational approach described here is crucially different from the RKKY approximation often used in the theory of spin glasses. ${ }^{60}$ There the coupling of the carrier spin density to the local moments is treated as a perturbation to the nonpolarized carrier Fermi sea. This is justified provided that the free-carrier Fermi energy is large compared to the energy scale of the exchange coupling which is conveniently measured in terms of the carrier mean-field splitting $\Delta=J_{p d} S N_{M n}$, where $N_{M n}$ is the density of $\mathrm{Mn}$ spins. However, for typical parameters of (III,Mn)V systems the Fermi energy and $\Delta$ are fairly of the same order of magnitude with the former quantity being often even smaller. Therefore the free-carrier ground state is not a good starting point for perturbation theory. To explore the true ground state of the system described by the Hamiltonian (1) for parameters realistic for (III,Mn)V semiconductors one should rather use the carrier ground state in the presence of a fully polarized Mn spin system as the starting point, as done here.

\section{INSTABILITY OF THE COLLINEAR FERROMAGNETIC STATE IN THE ISOTROPIC PARABOLIC-BAND MODEL}

\section{A. General discussion of the energy gradient}

As already stressed in Sec. II B, the expression (11) obtained for the ground-state energy in lowest order in the Holstein-Primakoff variables is very general; i.e., it is formally the same for any representation of the carrier system (e.g., parabolic band, six-band $\vec{k} \cdot \vec{p}$ Hamiltonian, etc.).

Let us now examine further the coefficients given in Eqs. (12) and (13) that enter the energy gradient (11). The imaginary part $g_{I}^{2}$ is nonzero if and only if the vector

$$
\vec{m}_{I}:=\int d^{3} r J\left(\vec{r}-\vec{R}_{I}\right)\langle\vec{s}(\vec{r})\rangle
$$

does not lie in the plane spanned by $\vec{e}_{\varphi_{I}}=\left(\cos \varphi_{I}, \sin \varphi_{I}, 0\right)$ and $\vec{e}_{z}=(0,0,1)$. Similarly, the real part $g_{I}^{1}$ is nonzero if and only if the projection of $\vec{m}_{I}$ onto the plane spanned by $\vec{e}_{\varphi_{I}}, \vec{e}_{z}$ is not collinear with the direction $\vec{\Omega}_{I}$ $=\left(\sin \vartheta_{I} \cos \varphi_{I}, \sin \vartheta_{I} \sin \varphi_{I}, \cos \vartheta_{I}\right)$ of the Mn spin at site $I$. In summary, for a given orientation of the Mn local moments to be stationary in energy the local directions $\vec{\Omega}_{I}$ must be parallel or antiparallel with $\vec{m}_{I}$ at each Mn site $I$. An example for such a situation is the collinear ferromagnetic state with all Mn spins in parallel in a simple parabolic-band model. Here the spins of all carrier eigenstates are polarized along the common axis of the Mn spins, and therefore $\vec{m}_{I}$ is always collinear with this direction at each site $I$. Thus, this state is always stationary, but as we shall see below, in general not stable. Moreover, the collinear ferromagnetic state turns out to be not even stationary if anisotropy induced by spin-orbit interactions is present, as is the case for valence-band holes in III-V semiconductors.

\section{B. Numerical results}

We now study the stability of the collinear ferromagnetic state in a simple parabolic-band model where the carriers are characterized by just one effective mass $m^{*}$. As seen before, the energy gradient vanishes for such a state, and the quadratic zero-frequency $(n=0)$ contribution to the effective action (14) describes the energy of static fluctuations around the collinear state. Thus, for this state to be stable, the matrix $D_{I J}^{-1}(0)$ must have non-negative eigenvalues only, while the occurrence of negative eigenvalues of this matrix indicates that the perfectly collinear state is not the ground state. We note that for any arrangement of the Mn positions $R_{I}$ the matrix $D_{I J}^{-1}(0)$ contains a zero eigenvalue corresponding to a uniform rotation of all spins. If $D_{I J}^{-1}(0)$ is non-negative, its eigenvalue distribution can be interpreted as a density of states (DOS) for magnetic excitations above the collinear state. 


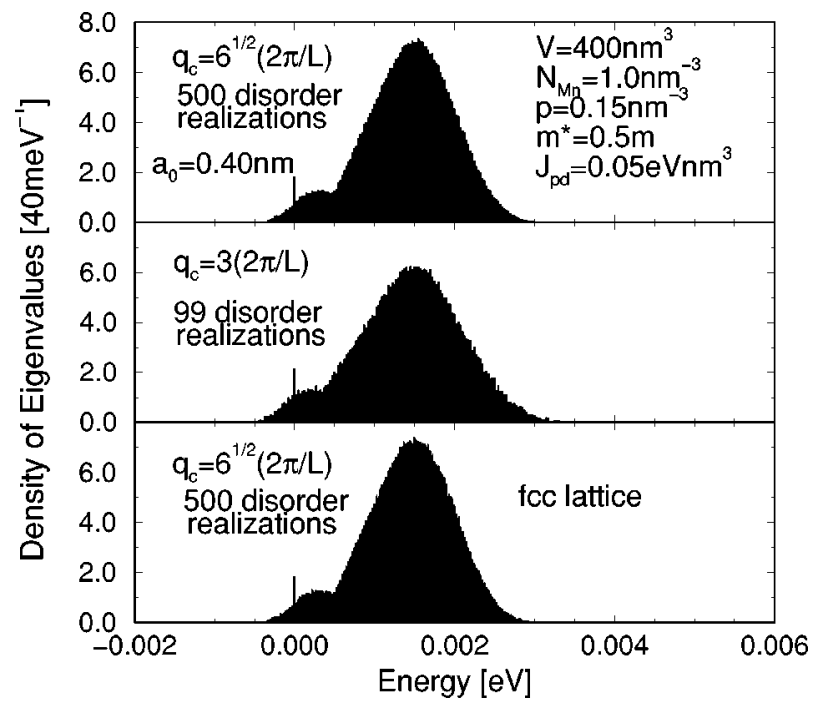

FIG. 1. The disorder-averaged density of states of magnetic excitations for a simulation cube of volume $V=L^{3}=400 \mathrm{~nm}^{3}$ with a Mn density of $N_{M n}=1.0 \mathrm{~nm}^{-3}$ and a density of $p=0.15 \mathrm{~nm}^{-3}$ of carriers having a band mass of half the bare electron mass. The strength of the exchange interaction between ions and carriers is $J_{p d}=0.05 \mathrm{eV} \mathrm{nm}^{-3}$ with a spatial range of $a_{0}=0.40 \mathrm{~nm}$ The two upper panels show data for different wave vector cutoff $q_{c}$ with the Mn positions chosen completely at random. The lowest panel contains data for the same situation as the top one but with the Mn positions chosen from an fcc lattice. The peaks at zero energy are due to the uniform rotation mode which strictly occurs in any disorder realization.

We have evaluated the spectrum of $D_{I J}^{-1}(0)$ in systems given by a simulation cube with periodic boundary conditions averaging over different realizations of the Mn positions. The single-particle wave functions $\psi_{\alpha \sigma}(\vec{r})$ are computed in a plane-wave basis taking into account wave vectors $\vec{q}$ with length up to an appropriate cutoff $q_{c}$. The same truncated plane-wave basis is used to compute the quantities (18) entering Eq. (17). Note that, for fluctuations around the collinear ferromagnetic state, $D_{I J}^{-1}(i \omega)$ is always real and symmetric for real $\omega$ since all carrier wave functions have for a given spin projection $\sigma$ a coordinate-independent phase (and can therefore also chosen to be real). This follows from the fact that the single-particle Hamiltonian describes for each spin projection just the problem of a spinless particle in a potential landscape provided by the Mn ions. Since $D_{I J}^{-1}(i \omega)$ is real and symmetric, the components of each of its eigenvectors have all the same phase (and can be chosen to be real). Physically this corresponds to the invariance of the system under rotations around the magnetization axis of the collinear state.

The two upper panels of Fig. 1 show results for typical system parameters for two different values of $q_{c}$. The comparison of both panels shows that the effects of the wave vector cutoff on the low-lying excitations have already saturated for the smaller $q_{c}$. The by far largest contributions to the DOS lie at positive energies, while a small amount of sightly negative eigenvalues of $D_{I J}^{-1}(0)$ indicates an instability of the perfectly collinear state.

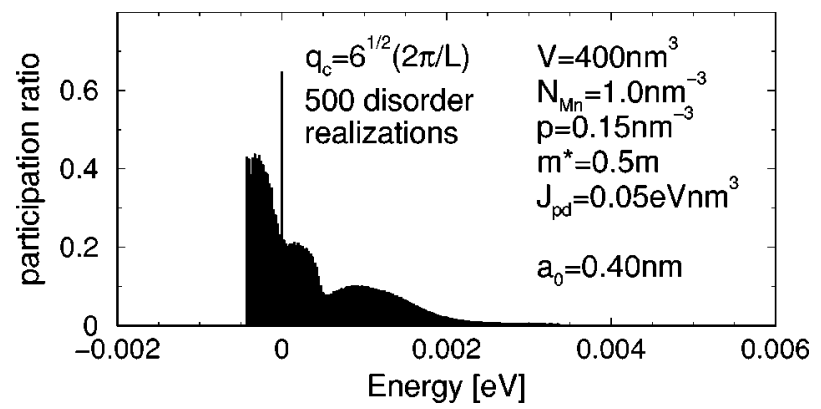

FIG. 2. The disorder-averaged participation ratio for the same situation as in the top panel of Fig. 1. The data are averaged over the sample intervals of the histogram. The value at zero energy is enhanced due to the contribution of the uniform rotation mode in that sample interval.

In the calculations discussed so far the Mn positions were chosen completely at random with uniform distribution, while in a real (III,Mn)V semiconductor the Mn ions are supposed to be located on the cation sites forming an fcc lattice. In the bottom panel of Fig. 1 we show data for the same system parameters as in the top panel but with the Mn positions chosen from an appropriate fcc lattice such that about $5 \%$ of all sites are occupied. Both plots are practically identical, indicating that our observations do not depend on this detail of the modeling.

The shape of the eigenvalue distribution of the fluctuation matrix $D_{I J}^{-1}(0)$ is quite sensitive in detail to the Mn density $N_{M n}$, the carrier density $p$, and the Hamiltonian parameters $m^{*}, J_{p d}$ and $a_{0}$. In our numerics, we have extensively investigated this high-dimensional parameter space in regions realistic for (III,Mn) V semiconductors. The general finding is that the ground state of the system is generically noncollinear. To our observation there occur always negative eigenvalues of $D_{I J}^{-1}(0)$, indicating the instability of the collinear state, provided that large enough wave vector cutoffs and system sizes are considered.

To analyze further the nature of this instability we consider the participation ratio

$$
p(E)=\left[N V \sum_{I}\left|\alpha_{I}(E)\right| 4\right]^{-1},
$$

where $\alpha_{I}(E)$ is the $I$ th component of the (normalized) eigenvector of $D_{I J}^{-1}(0)$ with eigenvalue $E$ and the summation goes over all $N V$ Mn sites. This quantity is an estimate for the fraction of components of $\alpha(E)$ being substantially nonzero. For instance, if a vector contains exactly a fraction of $p$ nonzero components of equal modulus, all others being zero, its participation ratio is $p$. The largest participation ratio of unity is achieved for the zero-energy uniform rotation mode where all components of the corresponding eigenvector are equal.

Figure 2 shows the disorder-averaged participation ratio for the same situation as in the top panel of Fig. 1. The negative-energy modes have clearly higher participation ratio than the eigenvectors at positive energy. This shows that the instability of collinear state is due to long-ranged dynamics 
involving a large fraction of the spins present in the system. Qualitatively the same observations are made for other values of system parameters.

\section{NONCOLLINEAR GROUND STATES AND THE INFLUENCE OF A MAGNETIC FIELD}

In the previous section we have investigated the stability of the collinear ferromagnetic state in the parabolic-band kinetic-exchange model. This state is always stationary (i.e., has a vanishing energy gradient) but not necessarily stable. In the present section we extend our ground-state studies using the energy gradient expression (11). We will consider the case of simple parabolic bands as well as more sophisticated kinetic carrier Hamiltonians incorporating spin-orbit anisotropy.

\section{A. Stationary states}

\section{Helical states in the parabolic-band model}

We now discuss a particular class of metastable states in the parabolic-band model.

The energy gradient expression (11) can be employed in a numerical steepest-descent procedure to search for true energy minima. To this end, one starts with the collinear state with all spins pointing into the $z$ direction and steps down in energy by performing sufficiently small rotations of the $\mathrm{Mn}$ spins according to some negative eigenvector of $D_{I J}^{-1}(0)$ (or some linear combination of them). Now consider the case that only one eigenvector is involved with all components having a certain common phase. The resulting orientations of Mn spins will all lie in the same plane spanned by the $z$ axis and a direction in the $x y$ plane that is determined by the above phase factor. Since the parabolic-band Hamiltonian is invariant under spin flips with respect to this given plane, the local spin density $\langle\vec{s}(\vec{r})\rangle$ and consequently the vectors $\vec{m}_{I}$ will also lie in this plane. ${ }^{61}$ As seen in the previous subsection, for such a situation the imaginary part $g_{I}^{2}$ of the coefficients vanishes identically for all $I$. When now applying the energy gradient expression in a steepest-decent procedure the real part $g_{I}^{1}$ of the gradient coefficients will just move the directions $\vec{\Omega}_{I}$ within this plane, while the imaginary parts $g_{I}^{2}$ remain strictly zero. Eventually this procedure will end up in a stationary state with all Mn spins lying in a plane prescribed by the initial departure from the collinear state.

These conclusions are confirmed by explicit numerics where such complanar (or helical) stationary states are indeed observed. This states can be seen as bona fide local minima on the energy landscape.

However, as explained in more detail in the following subsection, such a type of energy minima occurs only in isotropic models but not in systems with spin-orbit anisotropy.

\section{Nonstationarity of the collinear state in the presence of spin-orbit anisotropy}

We now examine the energy gradient (11) with respect to a six-band $\vec{k} \cdot \vec{p} \quad$ Hamiltonian involving spin-orbit anisotropy. ${ }^{26,54,55}$ In this case the rotational invariance in spin space is broken down to the cubic symmetry of the underlying GaAs crystal.

Let us first consider the collinear ferromagnetic state. In this case we find numerically that the vectors $\vec{m}_{I}$ are not parallel (or antiparallel) with any given common orientation of the Mn spins. This finding includes also the crystallographic symmetry axes $(1,0,0),(1,1,0)$, and $(1,1,1)$ or their equivalents.

There are two ways to restore the collinearity between the vectors $\vec{m}_{I}$ and a common orientation of the Mn spins.

(i) Virtual crystal or continuum approximation. If the $\mathrm{Mn}$ spins are approximated as an continuum and point all along one of the crystallographic symmetry axes $(1,0,0),(1,1,0)$, and $(1,1,1)$ or their equivalents, the $\vec{m}_{I}$ are always antiparallel to this direction. This finding explicitly confirms an assumption which earlier spin-wave calculations in the sixband continuum model were based on. ${ }^{27}$ Formally the continuum limit is reached by putting the parameter $a_{0}$ in the coupling function (2) to large values. In this limit the disorder with respect to the Mn positions is removed.

(ii) Zero spin-orbit coupling. For vanishing spin-orbit coupling the vectors $\vec{m}_{I}$ are antiparallel to any given common direction of the Mn spins.

Thus, in the presence of disorder with respect to the $\mathrm{Mn}$ positions and a finite anisotropy induced by the spin-orbit interaction, the collinear ferromagnetic is never stationary and therefore not the ground state. This statement includes the case that the common direction of the Mn spins is along some crystallographic symmetry axis.

This important result resembles recent findings by Zarand and Janko ${ }^{40}$ obtained within the RKKY approximation, where the ground state of the system was also found to be noncollinear (or, as termed there, frustrated).

Finally we briefly remark on the case that the directions of all Mn spins lie in some common plane. According to our numerics and as to be expected from the above results, the local spin density $\langle\vec{s}(\vec{r})\rangle$ and consequently the vectors $\vec{m}_{I}$ do not lie in plane of the Mn spins. Therefore truly complanar states being local energy minima do not exist if spin-orbit anisotropy is present

\section{B. Steepest-descent results and the role of an external field in the parabolic-band model}

We have employed the energy gradient expression (11) in a numerical steepest-descent procedure outlined in Sec. IV A 1 to search for stationary states in the parabolic-band model. These states can be considered as bona fide energy minima. Our results are as follows: In cases where the energy minimum found by this method is close to the collinear state (with a magnetization of about $90 \%$ of the maximum value or more), this minimum appears to be unique (for a given disorder realization) and can therefore be considered as the true absolute ground state of the system. However, in situations where the magnetization is reduced more substantially (by, say, about $20 \%$ or more) the energy minima found are not unique anymore but depend on technical details of 
the numerical procedure. In such cases the system is essentially spin-glass like with a complicated energy landscape. This situation occurs typically at larger density ratios $p / N_{M n}$ of carriers and Mn spins. For the system shown in Fig. 1 for instance the magnetization values in the energy minima typically found are of about $30 \%-40 \%$ of the collinear state.

Finally we consider the role of a magnetic field coupled to the Mn spins,

$$
\mathcal{H}_{B}=\vec{\Delta} \cdot \sum_{I} \vec{\Omega}_{I}
$$

where $\vec{\Delta}=g \mu_{B} S \vec{B}$ is the vector of Zeeman couplings. Here the magnetic field is coupled only to the Mn spins and not to the band carriers, since their mean-field spin splitting $\Delta$ $=J_{p d} S N_{M n}$ is large compared to the Zeeman couplings considered here. Therefore this contribution to the effective carrier spin splitting is negligible, and one should expect the collinear state to be stabilized by an external Zeeman field which is equal in magnitude to the smallest (negative) eigenvalue of the fluctuation matrix $D_{I J}^{-1}(0)$. For instance, from the data shown in Fig. 1 this Zeeman field would be about $0.5 \mathrm{meV}$.

A magnetic field in the $z$-direction leads to an additional contribution to the gradient components $g_{I}^{1}$ of $\Delta^{z} \sqrt{2 / S} \sin \vartheta_{I}$, which we shall consider in the following. We employ the following numerical procedure. Starting from the collinear state with all $\mathrm{Mn}$ spins pointing in the $z$ direction we obtain a noncollinear energy minimum by the steepest-descent method described above. Then we add a magnetic field in the $z$ direction and repeat the procedure to reach a new energy minimum. This state is then the new starting point for a steepest-descent walk with an increased magnetic field. By iterating this procedure we obtain a zero-temperature magnetization curve as a function of the external field.

Our numerical results are as follows: In cases where the zero-field ground-state magnetization is substantially reduced from its maximum value for the collinear state (i.e. in the "glassy" regime), the magnetization at finite field depends quantitatively on the step width that is used in increasing the field. Figure 3 shows magnetization data for the same system parameters as in the data before. As seen from there the Zeeman field needed to realign all spin along the $z$ direction is somewhat larger than the expected value of $\Delta^{z}$ $=0.5 \mathrm{meV}$, which is a typical hysteresis effect.

\section{THE SMALL-FREQUENCY DEPENDENCE OF THE ACTION KERNEL AND ADIABATIC DYNAMICS}

In Sec. III we studied the stability of the collinear ferromagnetic state within a parabolic-band model in terms of the static component of the effective action kernel $D_{I J}^{-1}$. Here we extend these analysis to the regime of small but finite Matsubara frequencies $\Omega_{n}$.

Expanding $K_{I J}\left(\Omega_{n}\right)$ around $\Omega_{n}=0$ one has

$$
K_{I J}\left(\Omega_{n}\right)=K_{I J}(0)+i \Omega_{n} K_{I J}^{\prime}+\cdots,
$$

with

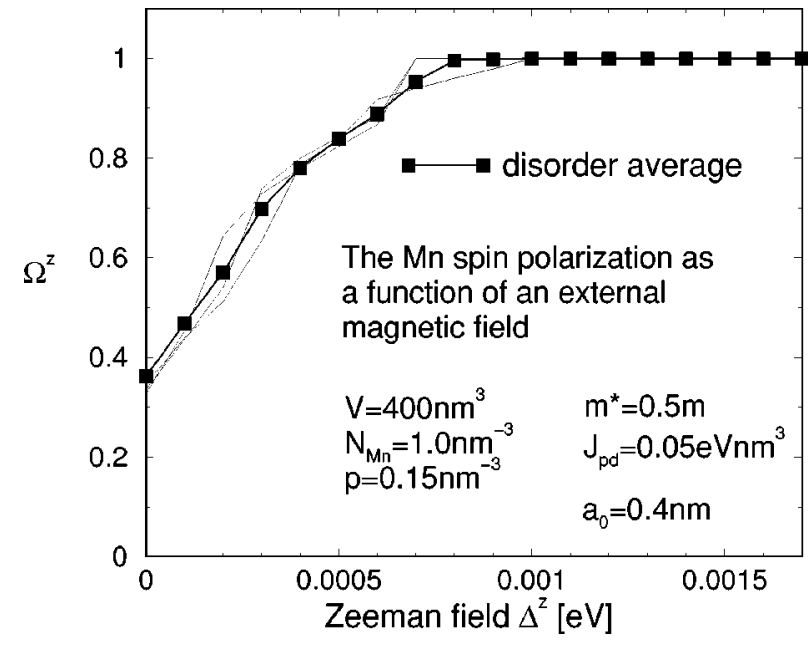

FIG. 3. The $z$ component of the $\mathrm{Mn}$ spin polarization $\Omega^{z}$ $=\Sigma_{I} \Omega_{I}^{z} /\left(N_{M n} V\right)$ as a function of an external magnetic field for the same parameters as in the top panel of Fig. 1. The Zeeman coupling is increased in units of $0.1 \mathrm{meV}$. The thin lines are results for three individual disorder realizations while the thick graph is the disorder average over 11 realizations.

$$
K_{I J}^{\prime}=-\frac{S}{2} \sum_{\alpha, \beta}\left[\frac{n_{F}\left(\eta_{\alpha}\right)-n_{F}\left(\eta_{\beta}\right)}{\left(\eta_{\alpha}-\eta_{\beta}\right)^{2}} F_{I}^{\alpha \downarrow, \beta \uparrow} F_{J}^{\beta \uparrow, \alpha \downarrow}\right] .
$$

The matrix $K^{\prime}$ fulfills an important sum rule which we derive now. As mentioned before, for the collinear state with all $\mathrm{Mn}$ spins pointing in the $z$ direction all carrier eigenstates have either spin up or spin down, and one ends up with two separated problems for spinless fermions in a potential landscape. The stationary Schrödinger equations for spin down and spin-up particles read

$$
\begin{aligned}
& -\frac{\hbar^{2} \nabla^{2}}{2 m^{*}} \psi_{\alpha \downarrow}(\vec{r})-\frac{S}{2} \sum_{I} \int d^{3} r J\left(\vec{r}-\vec{R}_{I}\right) \psi_{\alpha \downarrow}(\vec{r})=\varepsilon_{\alpha} \psi_{\alpha \downarrow}(\vec{r}), \\
& -\frac{\hbar^{2} \nabla^{2}}{2 m^{*}} \psi_{\beta \uparrow}(\vec{r})+\frac{S}{2} \sum_{I} \int d^{3} r J\left(\vec{r}-\vec{R}_{I}\right) \psi_{\beta \uparrow}(\vec{r})=\varepsilon_{\beta} \psi_{\beta \uparrow}(\vec{r}) .
\end{aligned}
$$

Taking all wave functions to be real and combining these equations one finds

$$
\left(\eta_{\beta}-\eta_{\alpha}\right)\left\langle\psi_{\alpha \downarrow} \mid \psi_{\beta \uparrow}\right\rangle=S \sum_{I} F_{I}^{\alpha \downarrow, \beta \uparrow}
$$

where $\langle\cdot \mid \cdot\rangle$ denotes a scalar product between spinless wave functions. Since both the spin-up and spin-down carrier wave functions fully span the Hilbert space of a single spinless particle, we have 


$$
\begin{aligned}
\sum_{\beta}\left\langle\psi_{\alpha \downarrow} \mid \psi_{\beta \uparrow}\right\rangle\left\langle\psi_{\beta \uparrow} \mid \psi_{\alpha \downarrow}\right\rangle & =\left\langle\psi_{\alpha \downarrow} \mid \psi_{\alpha \downarrow}\right\rangle=1 \\
& =\sum_{\beta} \frac{S^{2}}{\left(\eta_{\beta}-\eta_{\alpha}\right)^{2}} \sum_{I J} F_{I}^{\alpha \downarrow, \beta \uparrow} F_{J}^{\beta \uparrow, \alpha \downarrow}
\end{aligned}
$$

and similarly

$$
\sum_{\alpha} \frac{S^{2}}{\left(\eta_{\beta}-\eta_{\alpha}\right)^{2}} \sum_{I J} F_{I}^{\alpha \downarrow, \beta \uparrow} F_{J}^{\beta \uparrow, \alpha \downarrow}=1 .
$$

From these sum rules one derives ${ }^{62}$

$$
\sum_{I J} K_{I J}^{\prime}=\frac{1}{2 S}\left(n_{\uparrow}-n_{\downarrow}\right),
$$

where $n_{\uparrow}, n_{\downarrow}$ are the numbers of up- and down-spin carriers, respectively.

We now consider the limit of large inverse temperature $\beta$ where the bosonic Matsubara frequencies $\Omega_{n}=2 n \pi / \beta$ can be treated as a continuous variable $\Omega$. We are interested in the adiabatic limit of the spin dynamics which is described by the low-frequency limit of the effective action (14). In the expansion of the Fourier-transformed Holstein-Primakoff variables,

$$
z_{I}(\Omega)=z_{I}(0)+\Omega\left(\frac{d z_{I}}{d \Omega}\right)_{\Omega=0}+\cdots,
$$

the zero-frequency component $z_{I}(0)$ corresponds to Holstein-Primakoff variables constant in imaginary time $\tau$. Here we consider the uniform case $z_{I}(\tau)=\sqrt{S \eta} \exp (i \chi)$ for all $I$. This describes a uniform rotation of all Mn spins around the axis $(\sin \chi,-\cos \chi, 0)$ by an angle parametrized by $\eta$. As seen in Sec. III the matrix $D_{I J}^{-1}(0)$ annihilates the vector of the zero-frequency components $z_{I}(\Omega=0)$ $=\beta \sqrt{S \eta} \exp (i \chi)$. Therefore, using the sum rule (35), the low-frequency expansion of the effective action (14) reads

$$
S_{\text {fluc }}^{(2)}=\beta(i \Omega) \eta\left(-S N_{M n} V+\frac{1}{2}\left(n_{\uparrow}-n_{\downarrow}\right)\right)+\cdots,
$$

where $N_{M n} V$ is the number of Mn ions in the system. With an analytical continuation to real time, the above expression linear in $\Omega$ is the geometric phase generated by an adiabatic uniform rotation of all $\mathrm{Mn}$ spins (pointing initially along the $z$ direction) around an axis in the $x y$ plane by an angle $\beta \Omega \eta$. The second term in the parentheses stems from the carriers which adiabatically follow the Mn spins. Note that this contribution comes with a different sign. This is due to the fact that we are dealing with an effective action for the Mn spins only where the carriers have been integrated out, and therefore only the Mn spins are "actively" rotated. In our formalism the initial polarization axis of the Mn spins defines the quantization axis for the carrier spins, and rotating this axis is just a "passive" rotation of the carrier spin coordinate system. Therefore the geometric phase stemming from the carriers has a different sign.
We now turn to the case of general (i.e., nonuniform) adiabatic rotations of the Mn spins parametrized by other eigenvectors of $D_{I J}^{-1}(0)$ than the one discussed above. Then the geometric phase of the localized spins still stems from the frequency-dependent part of the diagonal matrix $L_{I J}(\Omega)$ [cf. Eq. (16)] while the largest contribution to the carrier phase will arise from $D_{I J}^{-1}(0)$ itself and the derivative $K_{I J}^{\prime}$. We therefore expect $K_{I J}^{\prime}$ to be dominantly diagonal when expressed in the eigenbasis of $D_{I J}^{-1}(0)$. In fact, this expectation is confirmed by numerical evaluations of this quantity. As a measure for the "diagonality" of a given matrix $A$ we consider

$$
D(A)=\left(\frac{\sum_{i} A_{i i}^{2}}{\operatorname{tr} A^{2}}\right)^{1 / 2} .
$$

This quantity is unity if $A$ is diagonal and of order the inverse of the square root of the dimension of $A$ if all of its elements are of the same order of magnitude.

We have evaluated the "diagonality" $D$ of $K_{I J}^{\prime}$ expressed in the eigenbasis of $D_{I J}^{-1}(0)$ for various sets of system parameters and averaged this quantity over many disorder realizations with respect to the Mn positions in space. We consistently find $D$ substantially larger than the average value of a general matrix. For instance, for the parameters used in Figs. 1 and 2 we find $\bar{D}=0.560$ (average over all disorder realizations) with a fluctuation $\Delta D=\sqrt{\bar{D}^{2}-\bar{D}^{2}}=0.045$. This is by an order of magnitude larger than the value of a general matrix of this dimension which is $D=1 / \sqrt{400}=0.05$.

\section{CONCLUSIONS}

We have studied the ground-state properties of kineticexchange models for carrier-induced ferromagnetism in (III,Mn)V semiconductors with randomly distributed Mn ions. Our method is embedded in a path-integral spin-wave-type formalism leading to an effective action for the Mn spins with full Matsubara frequency dependence. The zerofrequency contribution to this action is equivalent to static perturbation theory and characterizes the stability of a given spin configuration, while the component linear in frequency can be interpreted as the joint Berry phase of the $\mathrm{Mn}$ and carrier system.

Our perturbational approach to the ground state of the system studied here differs from the RKKY approximation insofar as we not do do perturbation theory around the freecarrier ground state but around the carrier ground state in the presence of a fully aligned collinear Mn spin system. This is appropriate since the mean-field spin splitting is for realistic system parameters not small compared to the Fermi energy. Therefore it cannot be regarded as a small perturbation to the free-carrier ground state, as done in the RKKY approximation. This approximation actually works well for metallic spin-glass systems where the coupling to local moments is indeed a small perturbation. ${ }^{60}$

For parabolic-band carriers the collinear ferromagnetic state with all Mn spins in parallel is always stationary but 
generically unstable. This instability can be characterized in terms of inverse participation ratios and is due to longranged nonlocal spin fluctuations. We also have presented results for the ground-state magnetization as a function of an external field.

For carrier dispersions involving anisotropy induced by spin-orbit coupling the collinear state is not even stationary and therefore also not the ground state. This interplay between the anisotropy in the carrier system and the disorder in the $\mathrm{Mn}$ positions reflects recent findings by Zarand and Janko $^{40}$ obtained within the RKKY approximation. The sta- tionarity of the collinear state is restored in the continuum or virtual crystal approximation where disorder is neglected.

\section{ACKNOWLEDGMENTS}

I thank A. L. Chudnovskiy, P. H. Dederichs, J. König, Q. Niu, F. von Oppen, D. Pfannkuche, N. Samarth, P. Schiffer, C. Timm, and, in particular, A. H. MacDonald for useful discussions. This work has been supported by the Swiss NSF, DARPA, ARO, the Indiana 21st Century fund, and the Welch Foundation.
${ }^{1}$ J. K. Furdyna and J. Kossut, Diluted Magnetic Semiconductors, Vol. 25 of Semiconductor and Semimetals (Academic Press, New York, 1988).

${ }^{2}$ H. Ohno, Science 281, 951 (1998).

${ }^{3}$ H. Ohno, J. Magn. Magn. Mater. 200, 110 (1999).

${ }^{4}$ H. Ohno and F. Matsukura, Solid State Commun. 117, 179 (2001).

${ }^{5}$ J. König, J. Schliemann, T. Jungwirth, and A. H. MacDonald, in Electronic Structure and Magnetism of Complex Materials, edited by D. J. Singh and D. A. Papaconstantopoulos (SpringerVerlag, Berlin, 2002).

${ }^{6}$ T. Dietl, Semicond. Sci. Technol. 17, 377 (2002).

${ }^{7}$ B. Lee, T. Jungwirth, and A. H. MacDonald, Semicond. Sci. Technol. 17, 393 (2002).

${ }^{8}$ S. Sanvito, G. Theurich, and N. A. Hill, J. Supercond. 15, 85 (2002).

${ }^{9}$ S. A. Wolf, D. D. Awschalom, R. A. Buhrman, J. M. Daughton, S. von Molnar, M. L. Roukes, A. Y. Chtchelkanova, and D. M. Treger, Science 294, 1488 (2001).

${ }^{10}$ Semiconductor Spintronics and Quantum Computation, edited by D. D. Awschalom, D. Loss, and N. Samarth (Springer, Berlin, 2002).

${ }^{11}$ H. Ohno, A. Shen, F. Matsukura, A. Oiwa, A. Endo, S. Katsumoto, and Y. Iye, Appl. Phys. Lett. 69, 363 (1996).

${ }^{12}$ M. L. Reed, N. A. El-Masry, H. H. Stadelmaier, M. K. Ritums, M. J. Reed, C. A. Parker, J. C. Roberts, and S. M. Bedair, Appl. Phys. Lett. 79, 3473 (2001).

${ }^{13}$ S. Sonoda, S. Shimizu, T. Sasaki, Y. Yamamoto, and H. Hori, J. Cryst. Growth 237, 1358 (2002).

${ }^{14}$ N. Theodoropoulou, A. F. Hebard, S. N. G. Chu, M. E. Overberg, C. R. Abernathy, S. J. Pearton, R. G. Wilson, and J. M. Zavada, Appl. Phys. Lett. 79, 3452 (2001).

${ }^{15}$ N. Theodoropoulou, A. F. Hebard, M. E. Overberg, C. R. Abernathy, S. J. Pearton, S. N. G. Chu, and R. G. Wilson, Phys. Rev. Lett. 89, 107203 (2002).

${ }^{16}$ Y. D. Park, A. T. Hanbicki, S. C. Erwin, C. S. Hellberg, J. M. Sullivan, J. E. Mattson, T. F. Ambrose, A. Wilson, G. Spanos, and B. T. Jonker, Science 295, 651 (2002).

${ }^{17}$ K. Ueda, H. Tabata, and T. Kawai, Appl. Phys. Lett. 79, 988 (2001).

${ }^{18}$ Y. Matsumoto, M. Murakami, T. Shono, T. Hasegawa, T. Fukumura, M. Kawasaki, P. Ahmet, T. Chikyow, S. Koshihara, and H. Koinuma, Science 291, 854 (2001).

${ }^{19}$ J. Okabayashi, T. Mizokawa, D. D. Sarma, A. Fujimori, T. Slu- pinski, A. Oiwa, and H. Munekata, Phys. Rev. B 65, 161203 (2002).

${ }^{20}$ T. Dietl, A. Haury, and Y. M. d'Aubigné, Phys. Rev. B 55, R3347 (1997).

${ }^{21}$ T. Dietl, H. Ohno, F. Matsukura, J. Cibert, and D. Ferrand, Science 287, 1019 (2000).

${ }^{22}$ T. Dietl, H. Ohno, and F. Matsukura, Phys. Rev. B 63, 195205 (2001).

${ }^{23}$ T. Jungwirth, W. A. Atkinson, B. H. Lee, and A. H. MacDonald, Phys. Rev. B 59, 9818 (1999).

${ }^{24}$ B. H. Lee, T. Jungwirth, and A. H. MacDonald, Phys. Rev. B 61, 15606 (2000).

${ }^{25}$ J. König, H. H. Lin, and A. H. MacDonald, Phys. Rev. Lett. 84, 5628 (2000); in Interacting Electrons in Nanostructures, edited by R. Haug and H. Schoeller (Springer, Berlin, 2001).

${ }^{26}$ M. Abolfath, J. Brum, T. Jungwirth, and A. H. MacDonald, Phys. Rev. B 63, 054418 (2001).

${ }^{27}$ J. König, T. Jungwirth, and A. H. MacDonald, Phys. Rev. B 64, 184423 (2001).

${ }^{28}$ J. Schliemann, J. König, H. H. Lin, and A. H. MacDonald, Appl. Phys. Lett. 78, 1550 (2001).

${ }^{29}$ J. Schliemann, J. König, and A. H. MacDonald, Phys. Rev. B 64, 165201 (2001).

${ }^{30}$ J. Schliemann and A. H. MacDonald, Phys. Rev. Lett. 88, 137201 (2002).

${ }^{31}$ T. Jungwirth, Q. Niu, and A. H. MacDonald, Phys. Rev. Lett. 88, 207208 (2002).

${ }^{32}$ S.-R. E. Yang and A. H. MacDonald, cond-mat/0202201 (unpublished); J. Sinova, T. Jungwirth, S.-R. E. Yang, J. Kucera, and A. H. MacDonald, Phys. Rev. B 66, 041202 (2002).

${ }^{33}$ R. R. dos Santos, L. E. Oliveira, and J. d'Albuquerque e Castro, J. Phys.: Condens. Matter 14, 3751 (2002).

${ }^{34}$ J. Fernandez-Rossier and L. J. Sham, Phys. Rev. B 64, 235323 (2001).

${ }^{35}$ I. Vurgaftman and J. R. Meyer, Phys. Rev. B 64, 245207 (2001).

${ }^{36}$ G. Bouzerar and T. P. Pareek, Phys. Rev. B 65, 153203 (2001); G. Bouzerar, J. Kudrnovsky, and P. Bruno, cond-mat/0208596 (unpublished).

${ }^{37}$ M. Berciu and R. N. Bhatt, Phys. Rev. Lett. 87, 107203 (2001); see, however, C. Timm, F. Schäfer, and F. von Oppen, cond-mat/0111504 (comment) Phys. Rev. Lett. (to be published); M. Berciu and R. N. Bhatt, cond-mat/0112165 (reply) Phys. Rev. Lett. (to be published). 
${ }^{38}$ M. P. Kennett, M. Berciu, and R. N. Bhatt, Phys. Rev. B 65, 115308 (2002).

${ }^{39}$ M. P. Kennett, M. Berciu, and R. N. Bhatt, Phys. Rev. B 66, 045207 (2002).

${ }^{40}$ G. Zarand and B. Janko, Phys. Rev. Lett. 89, 047201 (2002).

${ }^{41}$ A. L. Chudnovskiy and D. Pfannkuche, Phys. Rev. B 65, 165216 (2002).

${ }^{42}$ A. Kaminski and S. Das Sarma, Phys. Rev. Lett. 88, 247202 (2002).

${ }^{43}$ C. Timm, F. Schäfer, and F. von Oppen, Phys. Rev. Lett. 89, 137201 (2002).

${ }^{44}$ M. J. Calderon, G. Gomez-Santos, and L. Brey, Phys. Rev. B 66, 075218 (2002).

${ }^{45}$ M. Mayr, G. Alvarez, and E. Dagotto, Phys. Rev. B 65, 241202 (2002); G. Alvarez, M. Mayr, and E. Dagotto, Phys. Rev. Lett. 89, 277202 (2002).

${ }^{46}$ A. K. Bhattacharjee and C. Benoit à la Guillaume, Solid State Commun. 113, 17 (2000).

${ }^{47}$ H. Akai, Phys. Rev. Lett. 81, 3002 (1998).

${ }^{48}$ P. A. Korzhavyi, I. A. Abrikosov, E. A. Smirnova, L. Bergqvist, P. Mohn, R. Mathieu, P. Svedlindh, J. Sadowski, E. I. Isaev, Y. K. Vekilov, and O. Eriksson, Phys. Rev. Lett. 88, 187202 (2002).

${ }^{49}$ V. I. Litvinov and V. K. Dugaev, Phys. Rev. Lett. 86, 5593 (2001).

${ }^{50}$ T. Dietl, F. Matsukura, and H. Ohno, Phys. Rev. B 66, 033203 (2002).

${ }^{51}$ M. van Schilfgaarde and O. N. Mryasov, Phys. Rev. B 63, 233205 (2001).

${ }^{52}$ S. Sanvito, P. Ordejon, and N. A. Hill, Phys. Rev. B 63, 165206 (2001); S. Sanvito and N. A. Hill, Appl. Phys. Lett. 78, 3493 (2001)

${ }^{53}$ K. Sato and H. Katayama-Yoshida, Semicond. Sci. Technol. 17, 367 (2002).
${ }^{54}$ J. M. Luttinger and W. Kohn, Phys. Rev. 97, 869 (1955).

${ }^{55}$ W. W. Chow and S. W. Koch, Semiconductor-Laser Fundamentals (Springer, Berlin, 1999).

${ }^{56}$ S. J. Potashnik, K. C. Ku, S. H. Chun, J. J. Berry, N. Samarth, and P. Schiffer, Appl. Phys. Lett. 78, 1550 (2001); S. J. Potashnik, K. C. Ku, R. Mahendiran, S. H. Chun, R. F. Wang, N. Samarth, and P. Schiffer, Phys. Rev. B 66, 012408 (2002).

${ }^{57}$ G. M. Schott, W. Faschinger, and L. W. Molenkamp, Appl. Phys. Lett. 79, 1807 (2001).

${ }^{58}$ Note that the expression (20) has, just as $g_{I}$, dimension of energy, since the $z_{I}$ there are dimensionless. This is different from Eq. (11) where the quantities $z_{I}\left(\Omega_{n}\right)$ have the dimension of the inverse temperature such that $\mathcal{S}_{\text {fluc }}^{(1)}$ is dimensionless.

${ }^{59}$ We note that the summation over $\alpha$ and $\beta$ in Eq. (23) is unrestricted, i.e., is performed over all single-particle states. If one would restrict the summation to, say, states $\alpha$ that are occupied in the ground state and states $\beta$ that are unoccupied, a factor of 2 would occur.

${ }^{60}$ For a review see A. A. Abrikosov, Adv. Phys. 29, 869 (1980).

${ }^{61}$ If the spin flip is performed with respect to the $x z$ plane, the corresponding operator acting on the carrier states is just the operator $\mathcal{K}$ of complex conjugation which changes the sign of $\sigma^{y}$ but leaves the other Pauli matrices invariant. In the case of the $x y$ plane or $y z$ plane the spin-flip operator is $\sigma^{x} \mathcal{K}$ and $\sigma^{z} \mathcal{K}$, respectively. For a general orientation of this plane one has to use the appropriate linear combination of these operators. If all Mn spins lie in such a plane, the parabolic-band Hamiltonian is invariant under this spin-flip operation.

${ }^{62}$ Note that the sum rules, Eqs. (33) and (34), are independent of our choice to take all wave functions to be real since possible common phases in the up or down spins cancel in each term in the summation over $I, J$. 